\title{
A Collimation Scheme for Ions changing Charge State in the LEIR Ring
}

\author{
C. Bal, C. Carli, M. Chanel, E. Mahner, J.Pasternak $^{(1)}$ \\ CERN, Geneva, Switzerland
}

\begin{abstract}
Avalanche-like pressure rise and an associated decrease of the beam life-time, caused by (i) beam loss due to charge exchange interactions with rest gas molecules and (ii) electron capture from the electron beam of the electron cooler and (iii) ion impact induced outgassing, is a potential limitation for heavy ion accelerators. The vacuum system of the LEIR ring as to be upgraded to reach the dynamical vacuum pressure in the low $10^{-12}$ Torr range necessary to reach design performance. A collimation system to intercept lost ions by absorber blocks made of low beam-induced outgassing material will be installed. This paper reviews the collimation scheme and simulations of beam loss patterns around the ring.
\end{abstract}

1) Institute of Theoretical Physics, University of Wroclaw

Presented at PAC 2005, Knoxville, USA,

May 16-20, 2005

CERN,

CH-1211 Geneva 23,

Switzerland

Geneva, June 2005 


\title{
A COLLIMATION SCHEME FOR IONS CHANGING CHARGE STATE IN THE LEIR RING
}

\author{
J. Pasternak $\dagger \neq$, C. Bal $\dagger$, C. Carli $\dagger$, M. Chanel $\dagger$, E. Mahner $\dagger$ \\ †CERN, 1211 Geneva 23, Switzerland \\ $\ddagger$ Institute of Theoretical Physics, University of Wrocław, \\ pl. M. Borna 9, 50-204 Wrocław, Poland
}

\begin{abstract}
Avalanche-like pressure rise and an associated decrease of the beam life-time, caused by (i) beam loss due to charge exchange interactions with rest gas molecules, (ii) electron capture from the electron beam of the electron cooler and (iii) ion impact induced outgassing, is a potential limitation for heavy ion accelerators. The vacuum system of the LEIR ring has to be upgraded to reach the dynamical vacuum pressure in the low $10^{-12}$ Torr range necessary to reach design performance. A collimation system to intercept lost ions by absorber blocks made of low beam-induced outgassing material will be installed. This paper reviews the collimation scheme and simulations of beam loss patterns around the ring.
\end{abstract}

\section{THE LEIR RING}

The Low Energy Ion Ring (LEIR) at CERN (see Fig. 1) will accumulate $P b^{54+}$ ions coming from Linac 3 at 4.2 MeV/u. The aim is to generate high intensity and low emittance beams needed for the Large Hadron Collider (LHC) ion operation. The LEIR is being transformed from the former Low Energy Antiproton Ring (LEAR). A sophisticated muliturn injection and a fast electron cooling are the essential ingredients of the accumulation process, followed by bunching at $\mathrm{h}=2$ and acceleration to $72 \mathrm{MeV} / \mathrm{u}$ [1]. In order to extract an intensity of $9 \times 10^{8}$ ions every $3.6 \mathrm{~s}$, a sufficient beam life-time, long compared to accumulation is mandatory.

\section{MAIN MECHANISM OF ION LOSSES}

Ions in an accelerator can interact with residual gas molecules and, in particular, change charge state by electron capture or, for partially stripped ions, by electron loss. In the presence of electron cooling, ions can capture an electron directly from the electron beam of the cooler. In an accelerator lattice tuned to the nominal ion charge state $Q$, ions with charge state $Q+\Delta Q$ can be associated with an effective relative momentum offset:

$$
\frac{\Delta p_{e f f}}{p}=\frac{-\Delta Q}{Q+\Delta Q}\left(1+\frac{\Delta p}{p}\right)+\frac{\Delta p}{p},
$$

where $\frac{\Delta p}{p}$ denotes the relative momentum offset. Trans-

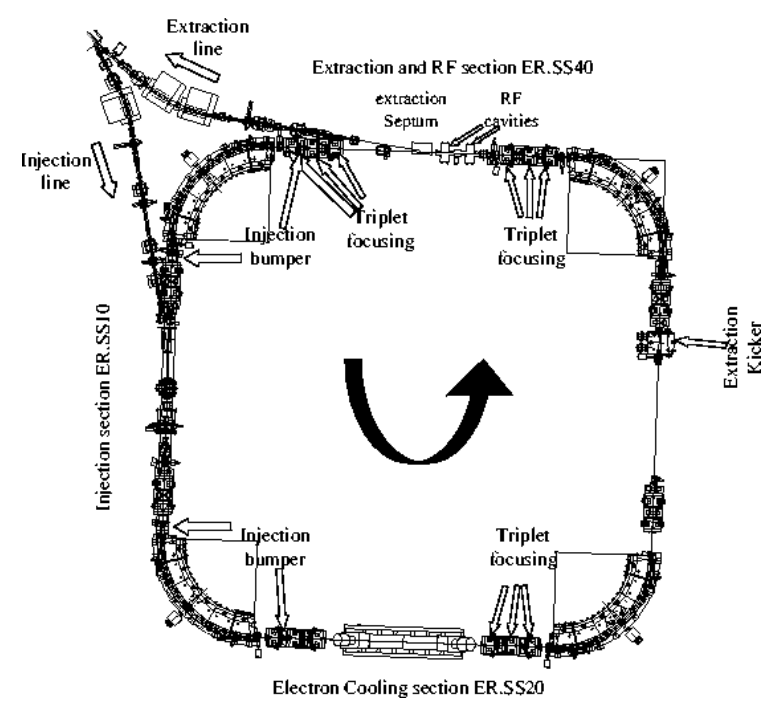

Figure 1: Layout of the LEIR ring.

verse equations of motion are the usual ones by taking $\frac{\Delta p_{e f f}}{p}$ instead of the relative momentum offset. The effective momentum offset of the ion after charge changing interaction exceeds usually the momentum acceptance of the machine.

\section{Avalanche Pressure Rise}

The following mechanism can lead to an avalanche-like pressure rise due to an ion beam in an accelerator:

- Ions change charge state due to interactions with rest gas molecules or with the electron beam of the cooler.

- Ions having changed charge state are lost downstream on the vacuum chamber wall.

- Lost ions release gas molecules from the vacuum chamber walls. In LEIR with stored $\mathrm{Pb}^{+54}$ at $4.2 \mathrm{MeV} / \mathrm{u}$ the desorption yield can be up to $2 \times 10^{4}$ molecules/ion [2] for the standard vacuum chamber (316LN stainless steel, treated by vacuum firing and baked in situ at $300^{\circ} \mathrm{C}$ ).

- Molecules released from the vacuum chamber increase the pressure and thus the loss rate due to charge exchange interactions. 
The process mentioned above depends on many details of the machine like pumping speed, distribution of vacuum pumps, details of the lattice design and the geometry and material of vacuum chamber used etc.

\section{Accumulation Tests in LEAR}

Many aspects of LEIR physics were tested during series of experiments performed between 1994 and 1997 in the LEAR ring [3]. In particular, during a proof of principle accumulation experiment in 1997, a dramatic decrease of the beam life-time to about $3 \mathrm{~s}$ was observed during accumulation. An associated pressure rise during continuous accumulation was observed as well. This showed, that an upgrade of the vacuum system in LEIR in comparison to the one used in LEAR is mandatory in order to reach the design performance.

\section{ION-INDUCED DESORPTION EXPERIMENTS}

In order to measure the ion-induced desorption yields of various types of vacuum chambers irradiated with 4.2 MeV/u lead ions, a dedicated experiment was installed in Linac 3 at CERN. The main results can be summarized as:

- The desorption yields of stainless steel vacuum chambers, coated with non-evaporable getter (TiZrV) or noble metals $(\mathrm{Au}, \mathrm{Ag}, \mathrm{Pd})$ are reduced by at least one to two orders of magnitude [4].

- The desorption yield depends on the ion impact angle and is about a factor of two smaller for perpendicular impact compared to grazing angle [2].

- All tested vacuum chamber materials show similar low desorption yields after long term $\mathrm{Pb}^{53+}$ ion bombardment [4].

\section{DISTRIBUTION OF LOSSES}

Loss patterns of ions undergoing charge changing interactions were calculated [5] assuming that ions are lost on the horizontal aperture (i.e. neglecting vertical betatron motion). This assumption is well justified in a machine with a vertical dispersion close to zero, which is the case for LEIR. Cross sections for electron capture are about one order of magnitude larger than those for electron loss $[6,7]$. Thus, we can focus on transitions of $\mathrm{Pb}^{54+}$ to $P b^{53+}$. The dynamics of $P b^{53+}$ in the lattice tuned for $\mathrm{Pb}^{54+}$ is described by a momentum deviation given by Eq. 1 of $1.88 \%$. Particles are distributed around the machine and tracked until the horizontal aperture is exceeded. The simulations were performed for ions created from the stack and the injected beam. Ions in the stack and in the injected beam were generated according to Gaussian and uniform distributions, respectively. The result can be summarized as following:
- Ions changing charge state in the straight sections are lost towards the end of the following bending section. This applies in particular to the ions capturing an electron from the electron cooler.

- Most ions undergoing charge exchange in the bending sections are lost either in the following straight section or towards the end of bending sections.

The distribution of losses in the nominal working point lattice $\left(Q_{x}, Q y\right)=(1.82,2.72)$ is shown in Fig. 2. The fact that losses are relatively localized is related to the rather long bending magnets, each one deflecting the beam by $90^{\circ}$. Loss patterns were calculated for different lattice settings yielding rather similar results.

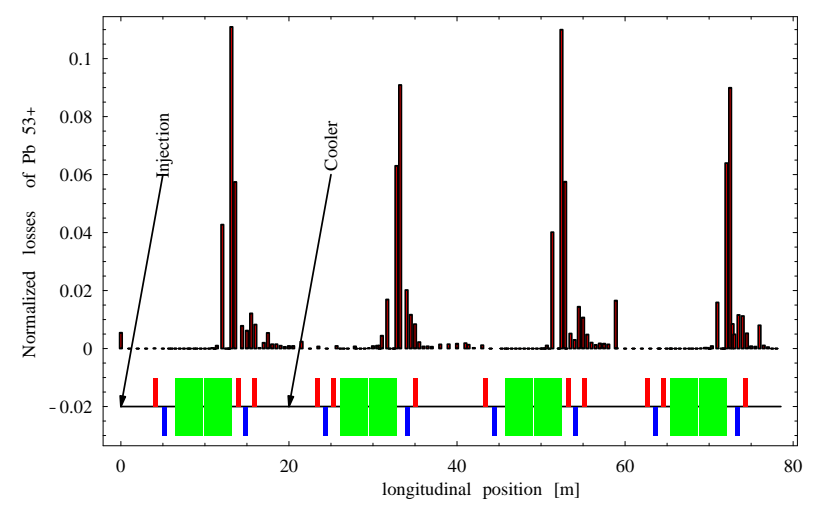

Figure 2: Loss pattern in the nominal working point lattice without collimators and assuming constant pressure around the ring.

\section{COLLIMATION SCHEME}

In order to efficiently intercept $P b^{53+}$ ions it was proposed to place two absorber blocks in every bending section, one at the position of the ion pump in the second half of the bending section and second towards the end of the bending. The collimators are mounted on the outward part of the vacuum chamber with respect to the machine center, as $P b^{53+}$ will be lost towards the outside of the ring due to positive dispersion. The collimators are stainless steel blocks coated in a galvanic bath with a $30 \mu \mathrm{m}$ thick gold film. The thickness was chosen to exceed the penetration depth of $4.2 \mathrm{MeV} / \mathrm{u}$ lead ions at normal impact, which is about $20 \mu \mathrm{m}$. The loss pattern in the nominal working point lattice with collimator is shown in Fig. 3 and corresponds to collimation efficiency of $81.4 \%$, calculated for collimators located $5 \mathrm{~cm}$ from the nominal charge beam axis. All collimators are installed in a way that enables a future exchange, if necessary.

\section{Movable Collimators}

In order to optimize the collimation efficiency and not to intercept circulating beam of $\mathrm{Pb}^{54+}$ in the presence of injection bump in various lattice optical configurations, the 


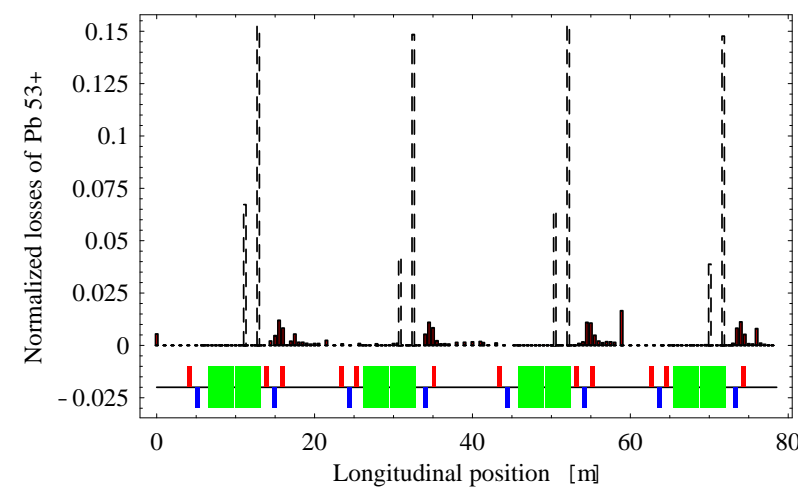

Figure 3: Loss pattern in the nominal working point lattice with collimators for the particles generated from the stack and assuming a constant pressure around the lattice. Ions hitting absorber blocks are presented by dashed histogram bars.

collimator located at the position of the ion pump will be equipped with a mechanical system, which enables to change its position with respect to the beam axis. The collimator can be moved by means of a stepping motor between -10 and $+40 \mathrm{~mm}$ with respect to its nominal position. The mechanical system of the collimator is separated from the flange by an intermediate titanium ring, which isolates thermally the motor and electronics from the vacuum chamber during the bake-out, due to very low thermal conductivity. The system is equipped with 2 end-switches and 2 mechanical end-stops, in case the microswitches fail. The technical $3 \mathrm{D}$ drawing of the movable collimator system is shown in Fig. 4. This gold coated stainless steel collimator has a size of $50 \times 50 \times 15 \mathrm{~mm}$.

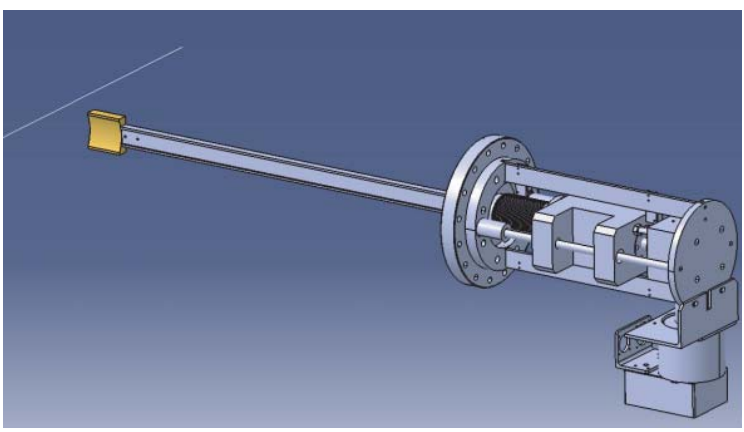

Figure 4: Drawing of the movable collimator system. The line on the left corresponds to the nominal $\mathrm{Pb}^{54+}$ ion beam.

\section{CONCLUSIONS}

The vacuum system of the LEIR ring is being upgraded to ensure a low dynamic vacuum corresponding to sufficiently long beam life-time to reach the design performance. In particular, the following measures are taken:

- Low-outgassing absorber blocks will be installed in the bending sections in order to intercept most ions lost due to charge changing interactions.

- Most lost ions not intercepted by absorbers hit the vacuum chamber in a straight sections. There, NEG coating will be applied wherever possible increasing substantially the pumping speed and, thus improving the dynamic vacuum.

If the measures described above are not sufficient, beam scrubbing may be applied. Beam will be injected continuously and will be lost on the vacuum chamber walls. The ion-induced pressure rise will decrease with time and the dynamic vacuum will improve.

\section{REFERENCES}

[1] Chapter 35 of Vol. III of the LHC design report.

[2] E. Mahner et al., PRST-AB 6, 013201 (2003).

[3] J. Bosser et al., Experimental Investigation of Electron Cooling and Stacking of Lead Ions in Low Energy Accumulation Ring, CERN/PS 99-033.

[4] E. Mahner et al., Ion Stimulated Gas Desorption Yields of Coated (Au, Ag, Pd) Stainless Steel Vacuum Chambers Irradiated with 4.2 MeV/u Lead Ions, CERN/AT 2003-006 (VAC).

[5] J. Pasternak, Loss Pattern of $\mathrm{Pb}$ Ions with Charge Changing Processes in the LEIR Ring, CERN-AB-Note-2004-066.

[6] B. Franzke, IEEE Trans. Nucl. Sci. NS-28, p. 2116 (1981).

[7] E. Mahner, CERN Report No. LHC/VAC-TN-2002-04 (2002). 